\title{
NARRATIVAS COMPARTIDAS EN EL HOGAR. UN ESTUDIO LONGITUDINAL DE LA ESTRUCTURA Y EL LENGUAJE EVALUATIVO
}

\section{SHARED NARRATIVES AT HOME. A LONGITUDINAL STUDY ABOUT THE NARRATIVE STRUCTURE AND THE EVALUATIVE LANGUAGE}

\author{
AlejandRa Stein ${ }^{*}$ \\ *Doctora en Ciencias del Lenguaje. Miembro de la Carrera del Investigador Científico del Consejo Nacional de \\ Investigaciones Científicas y Técnicas (CONICET) y docente de la Carrera de Ciencias de la Educación de la \\ Universidad de Buenos Aires (UBA). E-Mail: alejandrastein@yahoo.com.ar \\ Centro Interdisciplinario de Investigaciones en Psicología Matemática y Experimental Dr. Horacio J.A. Rimoldi (CIIPME). \\ Tte. Gral. Perón 2158, (C1040AAH) Ciudad Autónoma de Buenos Aires. República Argentina.
}

\section{REsumen}

El objetivo del trabajo que se informa fue estudiar la estructura y el lenguaje evaluativo en narrativas producidas por díadas niño-cuidador principal, durante el período comprendido entre los 2 años y 6 meses y 4 años. El corpus estuvo conformado por 81 narrativas producidas en distintos contextos conversacionales -situaciones de lectura de cuentos, conversaciones en torno a eventos pasados y conversaciones en torno a eventos futuros- en los que participaron en el hogar 6 niños pertenecientes a un grupo de sectores medios de Buenos Aires (Argentina). Se realizó un análisis de las narrativas con el fin de dar cuenta de su estructura y de los recursos evaluativos empleados por los niños y sus interlocutores. El estudio contempló también un análisis cuantitativo comparativo focalizado en la identificación de similitudes y diferencias en la estructura -atendiendo en particular el componente evaluativo en las mismas- y los recursos evaluativos entre las narrativas producidas longitudinalmente y en las diferentes situaciones (diferencia de medias y ANOVA). Los resultados mostraron el potencial de la elaboración de narrativas en colaboración para promover diferentes aspectos del desarrollo infantil, en particular aspectos relativos al desa- rrollo cognitivo y socioemocional de los niños. Tempranamente en la infancia y en los tres contextos estudiados los niños que participaron en el presente estudio elaboran junto con sus cuidadores, narrativas en donde se enfatiza la evaluación como componente estructural, esto es, la interpretación y el posicionamiento personal frente a los eventos.

Palabras clave: Estructura narrativa; Lenguaje evaluativo; Análisis longitudinal; Diferentes contextos conversacionales; Población de sectores medios.

\section{ABSTRACT}

The present study aims to analyze the structure and the evaluative language in narratives produced by child-caretaker dyads from Buenos Aires (Argentina), in different conversational contextsstorybook reading situations, conversations about past and future events- when children are 2 years and a half, 3 years, 3 years and a half and 4 years old.

Narrative is not only a type of discourse that organizes communicative exchanges and the transmission and recreation of culture; it is also 
one of the most important ways in which thought is configured (Bruner, 1986; Nelson, 1996; Rosemberg, Silva \& Stein, 2010). The narrative structure and the use of evaluative resources are particularly related to cognitive and socioemotional development (Fernández \& Melzi, 2008).

Narrative development begins during the preschool years (Nelson, 1996) and occurs through the social interactions that take place in diverse daily situations: play (Pellegrini, 1985), storybook reading (Snow, Porche, Tabors \& Harris, 2007) and conversations about past (Nelson, 1996) and future events (Hudson, 2002, 2006).

A series of studies undertaken in the fields of Psychology and Psycholinguistics have analyzed the structure and the evaluative language in early narratives orally produced by mother-child dyads (Fivush, 1991; Haden, Haine \& Fivush, 1997; Peterson \& McCabe, 1992, 1994, among others). The results of these studies have shown a relationship between the type of information introduced by mothers when they produce narratives in collaboration with their children and the information provided by children in the production of independent narratives later on (Fivush, 1991; Peterson \& McCabe, 1992, 1994). Other studies showed an increase of the information regarding actions as well as descriptive, orientative and evaluative components of the narratives as a function of children's age (Haden, Haine \& Fivush, 1997). The great majority of this research has been done with English-speaking and Caucasian families. Although there are some studies with Spanishspeaking population in Latin America (Díaz Oyarce \& Mendoza Saavedra, 2012; Fernández \& Melzi, 2008; Romero Contreras \& Gómez Martínez, 2013; Shiro, 2003) one can hypothesize there will be intra-group differences given the heterogeneity of Hispanic population.

There are few studies focused on the comparative analysis of narratives produced in different conversational contexts (Curenton, Craig \& Flanigan, 2008; Fernández \& Melzi, 2008). The author did not find studies which analyzed the evaluative resources used during the production of narratives about future events.

The corpus of the present study consists of 81 narratives produced in different conversational contexts: storybook reading situations (34 narratives), conversations about past events (24 narra- tives) and conversations about future events (23 narratives). The situations, which took place in the homes of 6 middle-income children from Buenos Aires (Argentina), were audio-recorded and transcribed for their analysis.

The narratives were analyzed regarding their structure and the evaluative resources used by the participants in order to answer the following questions:

What are the characteristics of the narratives collaboratively produced by children-caretakers dyads? In particular, which is the relative weight of the evaluative component regarding the other structural components referred to orientation, action and description?

Which evaluative resources are employed by the children and their interlocutors?

Are there any longitudinal and between conversational contexts differences regarding each of the aspects analyzed (structure of the narratives, evaluative component, evaluative resources)?

The results showed that the evaluative component of the narratives is the most predominant since 2:6 years and in the different conversational contexts considered. The children who participated in the study jointly produced with their caretaker (primarily their mothers) narratives where the interpretation and the personal stance on the events were emphasized. This fact reveals the potential of collaboratively produced narratives to promote different aspects of child development, particularly those related to cognition and socioemotional development.

Key words: Narrative structure; Evaluative language; Longitudinal analysis; Different conversational contexts; Middle-income families.

\section{INTRODUCCIÓN}

La narrativa constituye no solo un género discursivo que organiza los intercambios comunicativos, la transmisión y la recreación de la cultura, sino también una de las primeras y más importantes formas en que se configura el pensamiento (Bruner, 1986; Nelson, 1996; 
Rosemberg, Silva \& Stein, 2010). En efecto, diversos estudios han puesto de manifiesto la estrecha relación que existe entre narración, desarrollo lingüístico y cognitivo, y medio social y cultural. El desarrollo del discurso narrativo está ligado a la construcción de un sentido de sí mismo, a la conceptualización del tiempo, así como a la representación de uno mismo a lo largo del tiempo (Fivush \& Nelson, 2006; Hudson, 2002, 2006; Nelson, $1996,2007) \mathrm{y}$, en consecuencia, a la emergencia de la memoria autobiográfica (Nelson \& Fivush, 2004). Las narrativas tempranas se vinculan también con la socialización en una comunidad determinada (Aukrust \& Snow, 1998), con la organización, la comprensión y la reflexión sobre los eventos (Nelson, 2007), y con el desarrollo del lenguaje y la alfabetización (Beck, 2008; Snow, Porche, Tabors \& Harris, 2007).

Los inicios de este tipo de discurso tienen lugar durante los años preescolares través de la interacción social en situaciones cotidianas de juego (Pellegrini, 1985), lectura de cuentos (Snow et al., 2007) y conversaciones en torno a eventos pasados (Nelson, 1996; Fernández \& Melzi, 2008) y futuros (Hudson, 2002, 2006). En efecto, según Nelson (2007) y Tomasello $(1999,2003)$ el desarrollo del discurso narrativo tiene lugar a partir de un proceso de aprendizaje sociocultural que depende, por un lado, de la interacción con un patrón o estructura modelo -en este caso, de la narración- más avanzado que aquel desarrollado por el niño y, por otro, de la capacidad del niño para representar partes de ese modelo en la memoria; modelo al que puede acceder posteriormente en situaciones similares. En el marco de estos intercambios los niños comienzan a organizar e interpretar sus experiencias personales y las de otras personas, así como a anticipar y planificar eventos futuros. Inicialmente, son los adultos quienes proporcionan la mayor parte del contenido y la estructura de las primeras narrativas (Eisenberg, 1985), que integran información sobre las acciones implicadas en los eventos narrados, su ubicación temporal y espacial, la referencia a las personas involucradas, así como información acerca de la propia interpretación subjetiva de los eventos (Bamberg \& Damrad-Frye, 1991; Eisenberg, 1985; Haden, Haine \& Fivush, 1997; Newcombe \& Reese, 2004; Shiro, 2003).

Una serie de estudios realizados en el campo de la Psicología y la Psicolingüística analizaron la estructura de las primeras narrativas orales construidas en colaboración por niños pequeños y sus madres, dada su relevancia para el desarrollo infantil (Fivush, 1991; Peterson \& McCabe, 1992, 1994). Algunos de estos trabajos analizaron las variaciones longitudinales en el tipo y la cantidad de información incluida en los relatos, así como la relación entre la información proporcionada por los adultos tempranamente y la estructura de las narrativas producidas posteriormente por los niños de manera independiente. En esta línea, Fivush (1991) analizó la relación entre la provisión de información orientativa, referencial y evaluativa por parte de las madres en conversaciones sobre eventos pasados cuando los niños tenían 2 años y 6 años y la habilidad para producir narrativas independientes un año después. Los resultados de este estudio pusieron de manifiesto que aquellos niños cuyas madres proveían más información orientativa y evaluativa en las narrativas producidas en colaboración, incluían ese tipo de información en las narrativas independientes. De manera coincidente con estos hallazgos, Peterson y McCabe $(1992,1994)$ identificaron relaciones entre el tipo de información narrativa incluida por las madres en los intercambios tempranos a los 26 y 27 meses y las narrativas producidas por los niños a los 43 y 45 meses de edad.

Haden y colaboradores (1997) y Newcombe y Reese (2004) analizaron las variaciones longitudinales en la estructura de las narrativas producidas en colaboración por parte de díadas madre-niño norteamericanas y neozelandesas de habla inglesa. Haden y colaboradores (1997) observaron que tanto los niños como sus madres incrementaban la información sobre las acciones de los eventos, así como información referencial descriptiva, orientativa y evaluativa a lo largo del tiempo. Por su parte, Newcombe y Reese 
(2004) observaron que si bien los niños incrementaron el uso de evaluaciones y orientaciones, mostraban un estilo estable desde los 25 meses de edad.

Diversos trabajos han analizado en particular, el componente evaluativo de las narrativas tempranas producidas por niños pequeños y por díadas madre-niño en situaciones de lectura de cuentos y relatos de experiencias pasadas (Bamberg \& DamradFrye, 1991; Díaz-Oyarce \& Mendoza Saavedra, 2012; Ely, Gleason, Narasimhan \& McCabe, 1995; Fernández \& Melzi, 2008; Newcombe \& Reese, 2004; Peterson \& Biggs, 2001; Shiro, 2003). En conjunto, estos trabajos pusieron de manifiesto que alrededor de los 3 años de edad los niños emplean diversos procedimientos para transmitir la significación emocional del evento narrado y para concitar la atención de la audiencia. Según Labov (2001; Labov \& Waletzky, 1997) los procedimientos evaluativos suspenden o ralentizan la acción narrativa y, de ese modo, focalizan la atención en determinados aspectos de los eventos narrados. Estos procedimientos permiten cambiar el foco de la acción al narrador mediante la referencia explícita a eventos internos, el uso del discurso referido, así como recursos diversos destinados a intensificar, mitigar y realizar comparaciones entre distintos aspectos de los eventos narrados (Bamberg \& DamradFrye, 1991; Labov \& Waletzky, 1997; Peterson \& Biggs, 2001; Shiro, 2003).

Aquellos trabajos que analizaron variaciones en el lenguaje evaluativo según la edad de los niños, o bien se focalizaron en el estudio de recursos evaluativos específicos -la referencia a estados internos (Díaz Oyarce \& Mendoza Saavedra, 2012; Fernández \& Melzi, 2008; Shiro, 2003)-, o bien contemplaron diferentes grupos de edad en diseños comparativos transversales (Bamberg \& DamradFrye, 1991; Peterson \& Biggs, 2001; Romero Contreras \& Gómez Martínez, 2013). En esta línea, Fernández y Melzi (2008) observaron un incremento en la referencia a estados internos en conversaciones en torno a eventos pasados por parte de niños peruanos de 3 y 5 años y sus madres. Díaz-Oyarce y
Mendoza Saavedra (2012) identificaron únicamente diferencias en el empleo de claves de estado físico y relación en los relatos de ficción producidos de manera independiente por parte de niños chilenos de 5 y 6 años. Bamberg y Damrad-Frye (1991) y Peterson y Biggs (2001) analizaron, respectivamente, el empleo de recursos evaluativos durante la producción de narrativas de ficción y de experiencia personal en estudios comparativos transversales. El estudio de Bamberg y Damrad-Frye (1991) involucró a niños de 5 y 9 años y a estudiantes universitarios, en tanto que el de Peterson y Biggs (2001) incluyó a niños de 3, 5 y 8 años de edad. En ambos casos, los resultados mostraron diferencias en la cantidad de recursos evaluativos usados por el grupo de mayor edad en relación con los dos grupos menores, pero no entre los grupos de niños más pequeños entre sí.

La mayoría de los estudios mencionados fueron realizados con familias caucásicas de habla inglesa pertenecientes a sectores medios (Bamberg \& Damrad-Frye, 1991; Fivush, 1991; Haden et al., 1997; Newcombe \& Reese, 2004; Peterson \& Biggs, 2001; Peterson \& McCabe, 1992, 1994). Si bien, con menor frecuencia, se han llevado a cabo estudios con población hispana en algunos países de América Latina (Díaz Oyarce \& Mendoza Saavedra, 2012; Fernández \& Melzi, 2008; Romero Contreras \& Gómez Martínez, 2013; Shiro, 2003), cabe suponer la existencia de variaciones intragrupo dada la heterogeneidad de esta población. Asimismo, son escasos los estudios que analizaron comparativamente las narrativas producidas en diferentes contextos conversacionales (Curenton, Craig \& Flanigan, 2008; Fernández \& Melzi, 2008). No se identificaron estudios en los que se analizara el uso de recursos evaluativos en narrativas sobre eventos futuros.

En el marco de estos trabajos el objetivo del estudio que se informa consistió en el análisis transversal y longitudinal de las narrativas en colaboración producidas por niños pequeños ( 2 años y 6 meses a 4 años), pertenecientes a familias de sectores medios de Argentina, y sus cuidadores en contextos conversacionales de lectura de cuentos e in- 
tercambios acerca de eventos pasados y futuros.

Se atiende a la interrelación entre aspectos socioemocionales y cognitivos del desarrollo en la estructura de las narrativas y el uso de lenguaje evaluativo con el fin de responder a las siguientes preguntas de investigación:

¿Qué características presenta la estructura de las narrativas producidas en colaboración por los niños pequeños y sus interlocutores? En particular, ¿qué lugar ocupa el componente evaluativo de las narrativas en relación con aquellos referidos a la orientación, la acción y la descripción?

¿Qué recursos evaluativos emplean los niños y sus interlocutores?

¿Existen diferencias longitudinales y entre los contextos conversacionales en relación con cada uno de los aspectos analizados (estructura de las narrativas, componente evaluativo, recursos evaluativos)?

El análisis contempla el estudio comparativo de las narrativas de modo transversal (en distintos contextos conversacionales) y longitudinal (2 años y 6 meses, 3 años, 3 años y 6 meses y 4 años) con el fin de identificar eventuales similitudes y diferencias en la estructura y el lenguaje evaluativo.

\section{Metodología}

\section{CORPUS}

El corpus estuvo conformado por 81 narrativas producidas en situaciones de lectura de cuentos (34 narrativas), conversaciones en torno a eventos pasados (24 narrativas) y conversaciones en torno a eventos futuros (23 narrativas) en las que participaron en su hogar 6 niños pertenecientes a un grupo de sectores medios de Buenos Aires (Argentina), cuyos padres poseen educación terciaria o universitaria. Las situaciones fueron registradas cuando los niños tenían 2 años y 6 meses, 3 años, 3 años y 6 meses y 4 años de edad. El corpus específico de este estudio forma parte de una investigación más am- plia que contempla la administración de pruebas realizadas cuando los niños tenían 4 años y 6 meses y 5 años ${ }^{1}$.

OBTENCIÓN Y TRANSCRIPCIÓN DE LA INFORMACIÓN EMPÍRICA

Se realizó una visita cada seis meses al hogar de cada niño durante el período comprendido entre los 2 años y 6 meses y los 4 años de edad (4 visitas en total a cada hogar en el período considerado). En cada visita se indujeron las situaciones realizadas conjuntamente por el niño y un miembro del hogar a cargo de su cuidado: la lectura de un cuento (a partir de los 3 años y 6 meses se leyeron dos cuentos en cada visita), el relato de una experiencia pasada reciente y la conversación acerca de un evento futuro.

Con el objeto de intentar resguardar la validez ecológica de los datos no se pautó al interlocutor del niño (que podía ser el padre, la madre, hermanos u otra persona a cargo del niño habitualmente en el hogar). Tampoco se proporcionaron indicaciones relativas a la extensión de la situación ni a cómo interactuar con los niños. Asimismo, se les solicitó a las familias que registren por medio de audio una situación de comida luego de cada visita, con el objeto de contar con datos de interacciones entre el niño y los miembros de su hogar registrados sin la presencia de un observador.

Se tuvo acceso a las familias a través de contactos con personas conocidas. Con cada familia se estableció un acuerdo de anonimato y confidencialidad.

\footnotetext{
1 La investigación más amplia contempla también un estudio comparativo con niños pertenecientes a poblaciones urbano marginadas y una comunidad indígena qom de la Provincia de Buenos Aires. El corpus más amplio se encuentra en proceso de construcción. En el trabajo que se informa se analizaron las narrativas producidas en hogares de nivel socioeconómico medio recogidas hasta el momento de realizar este estudio.
} 
Para facilitar la comparabilidad de la información empírica, se controló el material de lectura -el cuento ilustrado-. Para la selección de los dos cuentos ilustrados utilizados se atendió especialmente a que los contenidos fueran familiares para los niños y que la historia tuviera una estructura canónica. Se optó por seleccionar libros con texto e imágenes debido a que son los que habitualmente se leen en los hogares de sectores medios de Argentina. Los títulos usados fueron: Ardilla tiene hambre (Kitamura, 1998) y El perro y el gato se ensucian (traducción de la autora de Cat and $\operatorname{dog}$ in a mess, Rayner, 2006).

Las situaciones fueron registradas por medio de audio y transcritas para su análisis. Los registros fueron complementados con las notas de campo elaboradas durante las observaciones y la autora llevó a cabo las transcripciones, realizando una desgrabación literal de los archivos de audio.

\section{Procedimientos PARA EL ANÁLISIS DE LOS DATOS}

Se analizaron narrativas ficcionales constituidas por la lectura del cuento ilustrado y narrativas no ficcionales sobre eventos pasados y futuros producidas en colaboración por los niños y sus madres (en algunos casos, estuvieron presentes también los hermanos y los padres de los niños). Para la identificación de las narrativas en las conversaciones en torno a eventos pasados y futuros se consideró a la narración como dos o más emisiones contiguas y temáticamente relacionadas, referidas a dos componentes cualesquiera de un evento desplazado en el tiempo pasado o futuro (Uccelli, 2009).

A continuación, se presentan tres fragmentos correspondientes a narrativas producidas durante la lectura de un cuento, una conversación en torno a un evento pasado y una conversación en torno a un evento futuro.

\section{1.- Lectura de cuento}

Ezequiel (4 años) y su madre leen un cuento. - Madre: (Lee) "Pero después el gato se en- sució todo". (Deja de leer). Mirá cómo está el gato. ¿Está contento?

- Niño: No.

- Madre: ¿Y por qué está tan enojado entonces?

(Silencio. La madre retoma la lectura)

- Madre: "El perro y el gato se revolcaron juntos en el barro”. (Deja de leer). Mirá, ahora sí. ¿Está contento ahora el gato? Tiene cara de contento. (Lee) "Y al final estaban los dos muuuyyyy sucios".

\section{2.- Conversación en torno a un evento pasado}

Mariana ( 3 años y 6 meses) y sus padres conversan acerca de un evento pasado.

- Madre: ¿Viste lo que nos pasó con el cochecito recién?

- Padre: Nos olvidamos el cochecito abajo del auto cuando estábamos en lo de la abu [abuela] y nos veníamos para acá.

- Madre: Nos subimos todos al auto y el cochecito quedó ahí.

- Padre: El cochecito de Vale (hermana de Mariana) quedó abajo. Por suerte lo encontró una señora y preguntó de quién era. Y el tío Juan dijo que era de su sobrina.

- Madre: ¡Mirá si perdíamos el cochecito!

- Niña: Mirá si alguien se lo robaba.

- Padre: ¿Viste? Por suerte hay gente honesta todavía.

- Madre: Hay gente buena que devuelve las cosas que no son de él o de ella. Vale se iba a quedar sin cochecito.

3.- Conversación en torno a un evento futuro

Juan (3 años) y su madre conversan acerca de un paseo al teatro que realizarán el fin de semana.

- Niño: Vamos a sentarnos primero (en el teatro). Después decimos que empiece, que empiece.

- Madre: Y empieza ¿y...?

- Niño: Y después no sé qué hace.

- Madre: (El artista) va a cantar canciones y todo eso divertido.

- Niño: Y después cuando termine no voy a llorar. Porque tengo tres añitos y no lloro.

- Madre: Porque a vos ¿qué te pasaba antes? 
¿Por qué llorabas?

- Niño: Porque quería que empiece porque me gustaba.

- Madre: No querías que termine porque te gustaban mucho las obras, ¿no? Y ahora ya sabés que sos grande, entendiste que termina y que otro día podés volver a verla, ¿no? Bueno entonces cuando termine no vas a llorar. ¿Y qué vas a hacer?

Se llevó a cabo un análisis de las narrativas producidas en colaboración por los niños y sus interlocutores con el fin de dar cuenta de su estructura y de los recursos evaluativos empleados por los participantes. En las conversaciones en torno a eventos pasados y futuros se codificaron todas las unidades terminales (Hunt, 1970, citado en Véliz, 1988) producidas por los niños y los otros participantes en el intercambio. En las situaciones de lectura de cuentos se codificaron únicamente aquellas que implicaban una modificación del texto original. La unidad terminal consiste en una cláusula principal más cualquier cláusula o cláusulas subordinadas agregadas a ella.

Para el análisis de las narrativas se emplearon de modo heurístico categorías desarrolladas en estudios previos referidas a la estructura de las narrativas (Haden et al., 1997; Newcombe \& Reese, 2004) y a los procedimientos evaluativos (Bamberg \& DamradFrye, 1991; Fernández \& Melzi, 2008; Labov \& Waletzky, 1967; Peterson \& Biggs, 2001). Las categorías desarrolladas en las investigaciones antecedentes se tomaron como base para el análisis de las narrativas analizadas en el estudio que se informa. Dichas categorías fueron modificadas con el fin de que las mismas se ajustaran y funcionaran adecuadamente en el corpus analizado (Vasilachis de Gialdino, 2006). En los Cuadros 1 y 2 se presentan las categorías, su definición y se ilustran con ejemplos tomados del corpus analizado de 81 narrativas.

El análisis contempló un estudio cuantitativo comparativo focalizado en la identificación de similitudes y diferencias en la estructura y los recursos evaluativos entre las narrativas producidas en las diferentes situa- ciones y entre cada uno de los momentos considerados ( 2 años y 6 meses, 3 años, 3 años y 6 meses y 4 años) (diferencia de medias y ANOVA).

Ejemplo:

Madre: Hay gente buena que devuelve las cosas que no son de él o de ella. [unidad terminal 1] / Vale se iba a quedar sin cochecito. [unidad terminal 2]

El presente fragmento tomado de una narrativa de evento pasado (ver ejemplo 2) contiene dos unidades terminales. Ambas corresponden al componente estructural evaluativo. En cada unidad se emplea un recurso evaluativo: gente buena (adverbios y adjetivos que manifiestan explícitamente la actitud del hablante) y se iba a quedar (negaciones, condicionales, futuros, y otras comparaciones, irrealis).

El análisis implicó la realización de los siguientes procesamientos:

1.- Se calculó la cantidad promedio de unidades terminales correspondiente a cada uno de los componentes estructurales de las narrativas.

2.- Se obtuvo un índice que analiza la cantidad de unidades terminales correspondientes al componente evaluativo en relación con la cantidad de unidades terminales correspondientes al resto de los componentes estructurales de las narrativas (evaluación / orientación + descripción + acción). Cuanto más se acerca a uno, más evaluada es la narrativa.

3.- Se calculó un índice que analiza la cantidad de información (medida en unidades terminales) referida a cada uno de los componentes estructurales aportada por el niño en relación con la cantidad de información referida a ese mismo componente, contenida en la narrativa construida en colaboración. Cuanto más se acerca a uno, mayor es la participación infantil.

4.- Se calculó la cantidad y la diversidad de recursos evaluativos usados por los niños y sus interlocutores.

5.- Se identificaron los recursos evaluativos más usados por los niños y sus interlocutores en la elaboración de las narrativas 
mediante el cálculo de frecuencias y porcentajes.

6.- Con la prueba ANOVA se analizaron las diferencias observadas en los procesamientos 1 a 4 entre los diferentes momentos y contextos conversacionales contemplados en el estudio para saber si eran significativas.

\section{Resultados}

\section{LA ESTRUCTURA NARRATIVA A LO LARGO DEL TIEMPO}

Los resultados pusieron de manifiesto escasas variaciones longitudinales en la estructura de las narrativas producidas en colaboración por los niños y sus madres. Como se muestra en el Gráfico 1, si bien se observó una disminución en la información referida a todos los componentes de las narrativas producidas entre los 2 años y 6 meses y los 4 años de edad, las diferencias solamente resultaron significativas en el caso del componente descriptivo, que disminuye de 4.9 unidades terminales por narrativa a los 2 años y 6 meses a 1.1 unidades terminales por narrativa a los 4 años (Prueba ANOVA de un factor: $F(3$, $76)=3.13 ; \mathrm{MSE}=16.9, p<.05$. Contraste de Bonferroni: diferencias entre los 2 años y 6 meses y los 4 años, $p<.05$ ). Se observó una mayor estabilidad a lo largo del tiempo en la cantidad de información referencial sobre las acciones de los eventos así como en la información evaluativa incluida en las narrativas.

Para estudiar en particular el componente evaluativo de las narrativas se calculó un índice que analiza la cantidad de unidades terminales correspondientes a dicho componente en relación con la cantidad de unidades terminales correspondientes al resto de los componentes estructurales de las narrativas bajo estudio (evaluación / descripción + orientación + acción). Asimismo, se contabilizó la cantidad de recursos evaluativos por narrativa empleados por parte de los niños y sus interlocutores en cada uno de los momentos contemplados en el estudio. Los resultados pusieron de manifiesto un incremento tanto en la proporción del componente evaluativo (.97 a los 2 años y 6 meses; 1.6 a los 3 años; 1.5 a los 3 años y 6 años y 1.4 a los 4 años) como en la cantidad de procedimientos evaluativos usados por los niños (ver Tabla 1) en las narrativas producidas a los 4 años ( 3 a los 2 años y 6 meses vs. 3.5 a los 4 años). Sin embargo, según la Prueba ANOVA de un factor, las diferencias no son estadísticamente significativas.

En todos los momentos contemplados, los recursos evaluativos más utilizados por los niños son los verbos y las frases verbales referidas a eventos internos, y los irrealis (Labov, 1972), esto es, el uso de negaciones, condicionales, futuros y otras comparaciones tales como los superlativos y las frases preposicionales con como. A partir de los 3 años los niños comienzan a emplear frecuentemente el conector causal porque para introducir explicaciones (ver Tabla 2). Por su parte, los interlocutores de los pequeños utilizan también con frecuencia la referencia a eventos internos emocionales, mentales y fisiológicos y los irrealis cuando los niños tienen 4 años de edad (ver Tabla 3).

\section{LA ESTRUCTURA NARRATIVA EN DIFERENTES CON- TEXTOS CONVERSACIONALES}

El análisis de la estructura de las narrativas producidas en las situaciones de lectura de cuentos y en las conversaciones en torno a eventos pasados y futuros puso de manifiesto que la información evaluativa es la predominante en cada uno de los contextos conversacionales considerados $(6.2 ; 13$ y 13.6 unidades terminales por narrativa respectivamente). Sin embargo, se observaron diferencias significativas en la estructura de las narrativas producidas en las situaciones de lectura de cuento y en las conversaciones en torno a eventos pasados y futuros, pero no entre estos dos últimos contextos entre sí. Como se observa en el Gráfico 2, las narrativas producidas en las conversaciones en torno a eventos pasados y futuros son similares en cuanto a la cantidad de información descriptiva (pasado: 4.3; futuro: 4), orientativa (pasado: 8.3 ; futuro: 7.6 ) y evaluativa (pasado: 13; futuro: 13.6).

Con el objetivo de analizar la participación infantil en cada uno de los contextos considerados se calculó un índice que analiza la 
cantidad de información referida a cada uno de los componentes estructurales aportada por los niños (medida en unidades terminales) en relación con la cantidad total de información referida a ese componente, contenida en las narrativas (ver Tabla 4). Los índices muestran una mayor participación de los niños en las conversaciones en torno a eventos pasados y futuros quienes, en dichos contextos, aportan entre un .2 y un .38 de la información descriptiva, orientativa y referida a las acciones de los eventos narrados. En cuanto al aporte de información evaluativa por parte de los niños, se observó que es mayor en las situaciones de lectura de cuentos (.26) y en las conversaciones en torno a eventos pasados (.36) en comparación con las conversaciones en torno a eventos futuros (.17). Los resultados de la prueba ANOVA de un factor muestran diferencias significativas en la participación de los niños en los diferentes contextos conversacionales.

El análisis cuantitativo de los procedimientos evaluativos puso de manifiesto que tanto los niños como sus interlocutores emplean una mayor cantidad y diversidad de recursos para evaluar los eventos narrados en las conversaciones en torno a eventos pasados y futuros. De acuerdo con la prueba ANOVA de un factor las diferencias observadas son estadísticamente significativas (ver Tablas 5 y 6).

En cuanto a los recursos evaluativos utilizados en cada uno de los contextos conversacionales estudiados, en todos los casos aquellos usados con mayor frecuencia son la referencia a estados internos y los irrealis (Labov, 1972). En las situaciones de lectura de cuento, los niños emplean con frecuencia conectores causales para introducir explicaciones (ver Tablas 7 y 8 ).

\section{DIscusión}

Los resultados del estudio muestran en conjunto, el potencial de la elaboración de narrativas en colaboración para promover diferentes aspectos del desarrollo infantil, en particular aspectos relativos al desarrollo cognitivo y socioemocional de los niños. Tempra- namente en la infancia y en los tres contextos estudiados los niños que participaron en el estudio elaboran junto con sus cuidadores narrativas en donde se enfatiza la interpretación y el posicionamiento personal frente a los eventos. En efecto, los resultados mostraron que el componente evaluativo es aquel que predomina en la estructura de las narrativas producidas en los cuatro momentos $(2$ años y 6 meses, 3 años, 3 años y 6 meses y 4 años) y en los tres contextos conversacionales analizados. Ello coincide con los hallazgos de estudios previos en los que se observó que alrededor de los 3 años de edad los niños recurren a distintos procedimientos para transmitir el tono subjetivo y emocional de los eventos narrados (Bamberg \& DamradFrye, 1991; Bamberg \& Reilly, 1994; Ely et al., 1995; Miller \& Sperry, 1988).

Si bien no se identificaron diferencias longitudinales significativas en cuanto a la cantidad de información evaluativa incluida en las narrativas, se observó al igual que en otros estudios (Bamberg \& Damrad-Frye, 1991; Díaz Oyarce \& Mendoza Saavedra, 2012; Shiro, 2003) una tendencia a un aumento de este componente narrativo reflejado en un incremento en la proporción de información evaluativa, la cantidad de recursos evaluativos empleados por los niños a los 4 años y el empleo de nuevos recursos evaluativos por parte de los niños, quienes a los 3 años comienzan a utilizar conectores causales para introducir explicaciones.

Como se señaló en la Introducción, la mayoría de los estudios previos en los que se comparó el uso de procedimientos evaluativos en niños de diferentes edades, contemplaron rangos de edad más amplios en estudios con un diseño comparativo transversal (Bamberg \& Damrad-Frye, 1991; Peterson \& Biggs, 2001; Romero Contreras \& Gómez Martínez, 2013). En este sentido, será importante incluir en futuros análisis las narrativas producidas en colaboración cuando los niños tienen 4 años y 6 meses y 5 años, una vez finalizada la construcción del corpus de datos del estudio más amplio.

Respecto de la comparación de la estructura y el lenguaje evaluativo en las narrativas 
producidas en distintos contextos conversacionales, se observaron mayores diferencias entre las narrativas producidas en situaciones de lectura de cuentos y los otros dos contextos estudiados -conversaciones en torno a eventos pasados y futuros-. Los relatos sobre experiencias pasadas presentan una estructura similar en cuanto a la cantidad de información descriptiva, orientativa y evaluativa, así como una mayor cantidad y diversidad de recursos evaluativos en comparación con las situaciones de lectura de cuentos. Si bien las narrativas sobre pasado contienen mayor información referencial sobre las acciones de los eventos que las narrativas sobre futuro, las diferencias no son estadísticamente significativas.

El análisis de las similitudes y diferencias en la estructura y el lenguaje evaluativo de las narrativas estudiadas revela la especificidad de cada contexto conversacional así como su potencial para promover el desarrollo lingüístico, cognitivo y socioemocional de los niños. En este sentido, se observó que las lecturas de cuento promueven la reflexión sobre los eventos internos de otros -los personajes de la historia-, así como sobre las causas de los eventos. En efecto, los recursos evaluativos más usados por los niños y sus madres en este contexto son la referencia a eventos internos -pensamientos, sentimientos, estados fisiológicos- y los conectores causales.

Por su parte, las conversaciones en torno a eventos pasados parecen facilitar la participación infantil. En los intercambios en torno a experiencias pasadas los niños aportan entre un 27 y un $38 \%$ de la información referida a los diferentes componentes estructurales de las narrativas. En particular, se observó que los niños aportan más información evaluativa durante los intercambios en torno a eventos pasados y las situaciones de lectura de cuentos que en las conversaciones en torno a eventos futuros. Ello puede deberse al hecho de que en las situaciones de lectura, la narración escrita constituye una referencia compartida y las ilustraciones del cuento físicamente presentes pueden contribuir a la atención conjunta (Tomasello, 1999). En el relato de experiencias pasadas, si bien los participantes intercambian en torno a una experiencia previa que no se halla físicamente presente, pueden recurrir a sus representaciones sobre el evento experimentado. En cambio, en las conversaciones acerca de eventos futuros, no existe como referencia una experiencia previa específica para construir la representación lingüística de lo que se hará en el futuro. Ello implica un mayor desafío para la participación infantil. Por otro lado, las conversaciones en torno a eventos pasados parecen ser más habituales y familiares para los niños que las conversaciones en torno a eventos futuros en los hogares de sectores medios de Argentina (Arrúe, 2012).

Sin embargo, es importante señalar que en las conversaciones analizadas en torno a eventos futuros, los niños y sus madres no se limitan a enumerar lo que harán o sucederá, sino que hipotetizan acerca de lo que querrían hacer y lo que podría o no suceder. Ello se refleja en el uso de irrealis (Labov, 1972) -negaciones, condicionales, comparaciones-. Estos hallazgos coinciden con los resultados de los estudios llevados a cabo por Hudson (2002, 2006) que, si bien no se focalizaron en el uso de procedimientos evaluativos, mostraron un mayor uso de lenguaje hipotético en este contexto en comparación con las conversaciones en torno a experiencias personales pasadas.

La evidencia empírica proporcionada por este estudio revela la particularidad y refuerza la potencialidad de los intercambios en torno a experiencias pasadas, eventos futuros y lecturas de cuentos como contextos relevantes no sólo para promover el desarrollo del discurso narrativo sino también el desarrollo lingüístico, cognitivo y socioemocional de los niños. Ello tiene implicancias educativas para el diseño de estrategias de intervención pedagógicas en el contexto escolar orientadas a promover dichos aspectos del desarrollo infantil durante los años preescolares.

En futuras investigaciones será importante profundizar estos análisis mediante el estudio de la organización interna de la información incluida en las narrativas así como de la función particular de los procedimientos evaluativos empleados por los niños y sus interlocutores. 


\section{CUADRO1}

COMPONENTES ESTRUCTURALES DE LAS NARRATIVAS

\begin{tabular}{|c|c|c|}
\hline Componente & Definición & Ejemplo \\
\hline Orientación & $\begin{array}{l}\text { Unidades referidas a información sobre } \\
\text { el espacio, el tiempo y las personas pre- } \\
\text { sentes en el evento. }\end{array}$ & Juan estaba en la plaza. \\
\hline Acción & $\begin{array}{l}\text { Unidades referidas a acciones que tu- } \\
\text { vieron, tienen o tendrán lugar en el } \\
\text { evento. }\end{array}$ & $\begin{array}{l}\text { Anduve en tractor. } \\
\text { Vamos a sacar fotos en el puerto. }\end{array}$ \\
\hline Evaluación & $\begin{array}{l}\text { Unidades que cumplen una función eva- } \\
\text { luativa, en tanto proveen información } \\
\text { acerca del tono subjetivo y emocional } \\
\text { del evento. }\end{array}$ & $\begin{array}{l}\text { Yo quiero llevar un juguete al campamento. } \\
\text { Las hormigas no son asco. } \\
\text { Si hay aguavivas no nos metemos en el } \\
\text { mar. }\end{array}$ \\
\hline Descripción & $\begin{array}{l}\text { Unidades que refieren a detalles des- } \\
\text { criptivos asociados a las acciones. }\end{array}$ & $\begin{array}{l}\text { Ese adornito, el de color rayado, lo pusiste } \\
\text { vos (en el árbol de Navidad). }\end{array}$ \\
\hline
\end{tabular}




\section{CUADRO 2}

Procedimientos eValuativos

\begin{tabular}{|c|c|c|}
\hline Recurso & Definición & Ejemplo \\
\hline $\begin{array}{l}\text { Verbos y frases verbales referidos a } \\
\text { eventos internos }\end{array}$ & $\begin{array}{l}\text { Referencias a eventos internos emo- } \\
\text { cionales, mentales y fisiológicos. }\end{array}$ & $\begin{array}{l}\text { Quería jugar con los baldes en la pla- } \\
\text { za. } \\
\text { Estaba cansado. }\end{array}$ \\
\hline $\begin{array}{l}\text { Negaciones, condicionales, futuros } \\
\text { y otras comparaciones (irrealis) } \\
\text { (Labov, 1972) }\end{array}$ & $\begin{array}{l}\text { Referencias a eventos que no suce- } \\
\text { dieron, podrían haber sucedido o } \\
\text { pueden suceder. Se evalúan los } \\
\text { eventos que efectivamente ocurrie- } \\
\text { ron mediante su comparación con } \\
\text { hechos potenciales o que no tuvie- } \\
\text { ron lugar. Se incluyen también su- } \\
\text { perlativos y frases preposicionales } \\
\text { con "como". }\end{array}$ & $\begin{array}{l}\text { Si no podemos sacarle fotos a los } \\
\text { lobos marinos le sacamos fotos a los } \\
\text { barcos. } \\
\text { Mirá si perdíamos el cochecito. }\end{array}$ \\
\hline Discurso referido & $\begin{array}{l}\text { Recursos mediante los que se atri- } \\
\text { buye y representa la palabra de las } \\
\text { personas / personajes involucrados } \\
\text { en el evento narrado. Comprende el } \\
\text { discurso referido directo e indirecto. }\end{array}$ & $\begin{array}{l}\text { Me gustó la animación que hiciste en } \\
\text { el cumpleaños. La tía también llamó } \\
\text { por teléfono y dijo: la animación que } \\
\text { hizo Felipe estuvo muy divertida. }\end{array}$ \\
\hline $\begin{array}{l}\text { Adverbios y adjetivos que manifies- } \\
\text { tan explícitamente la actitud del ha- } \\
\text { blante }\end{array}$ & $\begin{array}{l}\text { Adverbios y adjetivos que manifies- } \\
\text { tan de modo explícito la actitud del } \\
\text { hablante frente a aspectos específi- } \\
\text { cos o al evento narrado en general } \\
\text { (o en su globalidad). }\end{array}$ & $\begin{array}{l}\text { Estaba muy frío, estaba todo mo- } \\
\text { jado, ¿Estuvo divertido el cumplea- } \\
\text { ños?, Va a estar bueno que cante } \\
\text { esta canción Pescetti en el teatro. }\end{array}$ \\
\hline Conectores causales & $\begin{array}{l}\text { Conectores tales como porque, dado } \\
\text { que, puesto que, que introducen ex- } \\
\text { plicaciones. }\end{array}$ & Me enojé porque ella me pegó. \\
\hline Repetición & Repetición de palabras o ideas. & $\begin{array}{l}\text { Comí, comí y comí; Me quiso pegar, } \\
\text { Intentó pegarme. }\end{array}$ \\
\hline Elementos paralingüísticos & $\begin{array}{l}\text { Tono e inflexión de la voz, alarga- } \\
\text { miento de vocales, entre otros. }\end{array}$ & La roca estaba mmuuuyyyy pesada. \\
\hline
\end{tabular}




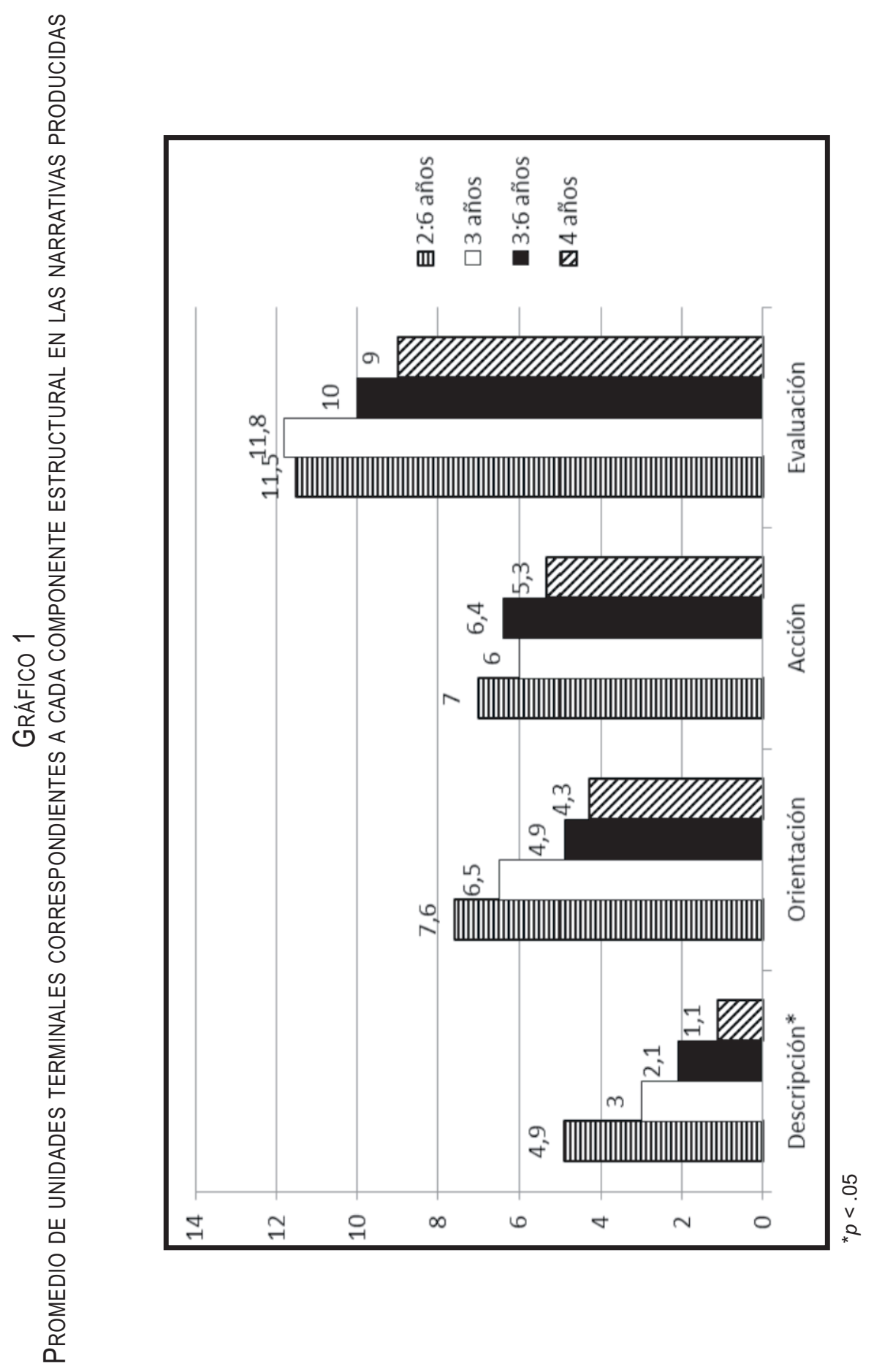



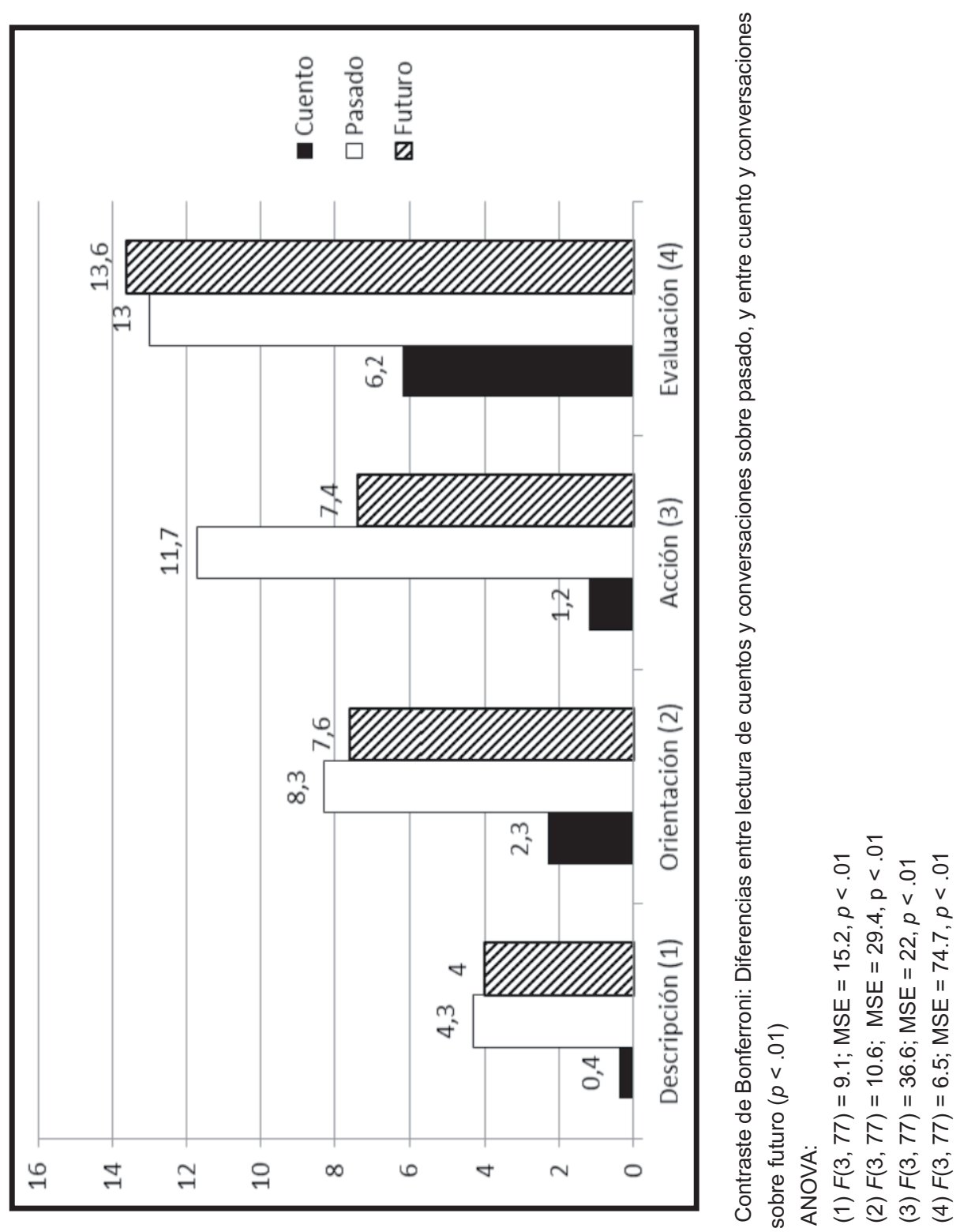
Narrativas compartidas en el hogar

TABLA 1

MEDIAS Y DESVIACIONES TíPICAS DE LOS RECURSOS EVALUATIVOS EMPLEADOS EN LAS NARRATIVAS PRODUCIDAS

\begin{tabular}{|c|c|c|c|c|c|c|c|c|}
\hline & \multicolumn{2}{|c|}{2 años y 6 meses } & \multicolumn{2}{|c|}{3 años } & \multicolumn{2}{|c|}{3 años y 6 meses } & \multicolumn{2}{|c|}{4 años } \\
\hline & $M$ & $D T$ & $M$ & $D T$ & $M$ & $D T$ & $M$ & $D T$ \\
\hline Niños & 3 & 2.4 & 4.2 & 5.2 & 3 & 3.5 & 3.5 & 5.2 \\
\hline Interlocutores & 9 & 5.6 & 10 & 9.6 & 8.6 & 9.7 & 7.8 & 7.8 \\
\hline Total & 12 & 6.9 & 14.2 & 12.7 & 11.6 & 12.5 & 11.3 & 11.9 \\
\hline
\end{tabular}

TABLA 2

FRECUENCIAS Y PORCENTAJES DE LOS RECURSOS EVALUATIVOS MÁS UTILIZADOS POR LOS NIÑOS EN LAS NARRATIVAS PRODUCIDAS

\begin{tabular}{|l|cc|cc|cc|cc|}
\hline \multicolumn{1}{|c|}{$\begin{array}{l}\text { Recursos más } \\
\text { utilizados }\end{array}$} & \multicolumn{2}{|c|}{2 años y 6 meses } & \multicolumn{2}{|c|}{3 años } & \multicolumn{2}{|c|}{3 años y 6 meses } & \multicolumn{2}{|c|}{4 años } \\
\hline Irrealis & $f$ & $\%$ & $f$ & $\%$ & $f$ & $\%$ & $f$ & $\%$ \\
Evento interno & 14 & 25 & 18 & 23 & 20 & 29 & 20 & 24 \\
Conector causal & 10 & 8 & 22 & 29 & 17 & 25 & 24 & 29 \\
\end{tabular}




\section{TABLA 3}

FRECUENCIAS Y PORCENTAJES DE LOS RECURSOS EVALUATIVOS MÁS UTILIZADOS POR LOS INTERLOCUTORES EN LAS NARRATIVAS PRODUCIDAS

\begin{tabular}{|c|c|c|c|c|c|c|c|c|}
\hline \multirow{2}{*}{$\begin{array}{c}\text { Recursos más } \\
\text { utilizados }\end{array}$} & \multicolumn{2}{|c|}{2 años y 6 meses } & \multicolumn{2}{|c|}{3 años } & \multicolumn{2}{|c|}{3 años y 6 meses } & \multicolumn{2}{|c|}{4 años } \\
\hline & $f$ & $\%$ & $f$ & $\%$ & $f$ & $\%$ & $f$ & $\%$ \\
\hline Irrealis & & & & & & & 50 & 27 \\
\hline Evento interno & 65 & 36 & 54 & 30 & 59 & 31 & 54 & 29 \\
\hline
\end{tabular}

TABLA 4

PROPORCIÓN DE APORTES REALIZADOS POR EL NIÑO EN LAS NARRATIVAS PRODUCIDAS SEGÚN EL CONTEXTO (INFORMACIÓN PROPORCIONADA POR EL NIÑO / TOTAL PARA CADA COMPONENTE ESTRUCTURAL)

\begin{tabular}{|c|c|c|c|c|c|c|c|c|}
\hline Contexto & $\begin{array}{l}\text { Cuentc } \\
\text { Proporción }\end{array}$ & $D T$ & $\begin{array}{l}\text { Pasado } \\
\text { Proporción }\end{array}$ & $D T$ & $\begin{array}{l}\text { Futuro } \\
\text { Proporción }\end{array}$ & $D T$ & & 77) \\
\hline Descripción & .01 & .40 & .27 & .26 & .33 & .31 & .05 & $p<.01$ \\
\hline Orientación & .15 & .24 & .38 & 15 & .34 & .23 & 9.1 & $p<.01$ \\
\hline Acción & .12 & .24 & .34 & .18 & .20 & .18 & 7.3 & $p<.01$ \\
\hline Evaluación & .26 & .29 & .36 & .19 & .17 & .16 & 3.7 & $p<.05$ \\
\hline
\end{tabular}

Contraste de Bonferroni: Diferencias entre Cuento y Pasado, y entre Cuento y Futuro $(p<.01)$ 
TABLA 5

MEDIAS Y DESVIACIONES TÍPICAS DE LOS RECURSOS EVALUATIVOS POR NARRATIVA EMPLEADOS POR LOS NIÑOS Y SUS INTERLOCUTORES EN LAS NARRATIVAS PRODUCIDAS EN DIFERENTES CONTEXTOS CONVERSACIONALES

\begin{tabular}{|c|c|c|c|c|c|c|c|c|}
\hline & \multicolumn{2}{|c|}{ Cuento } & \multicolumn{2}{|c|}{ Pasado } & \multicolumn{2}{|c|}{ Futuro } & \multirow{2}{*}{\multicolumn{2}{|c|}{$F(2,77)$}} \\
\hline & M & $D T$ & M & $D T$ & $M$ & $D T$ & & \\
\hline Niños & 2 & 2.6 & 5.2 & 4.8 & 3.6 & 4.7 & 4.5 & $p<.01$ \\
\hline Interlocutores & 5.1 & 6 & 8.8 & 6.4 & 13.7 & 10 & 8.6 & $p<.01$ \\
\hline Total & 7.1 & 7.7 & 14 & 9.8 & 17.3 & 13.9 & 7 & $p<.01$ \\
\hline
\end{tabular}

Contraste de Bonferroni: Diferencias entre Cuento y Pasado, y entre Cuento y Futuro $(p<.01)$

TABLA 6

DIVERSIDAD DE RECURSOS EVALUATIVOS POR NARRATIVA EMPLEADOS EN LAS NARRATIVAS PRODUCIDAS EN DIFERENTES CONTEXTOS CONVERSACIONALES

\begin{tabular}{|c|c|c|c|c|c|c|c|c|}
\hline & \multicolumn{2}{|c|}{ Cuento } & \multicolumn{2}{|c|}{ Pasado } & \multicolumn{2}{|c|}{ Futuro } & \multirow{2}{*}{\multicolumn{2}{|c|}{$F(2,78)$}} \\
\hline & M & $D T$ & M & $D T$ & $M$ & $D T$ & & \\
\hline Niños & 1.2 & 1.2 & 2.75 & 1.8 & 2 & 1.9 & 6.4 & $p<.01$ \\
\hline Interlocutores & 2.3 & 1.7 & 3.87 & 1.5 & 4.22 & 1.9 & 10 & $p<.01$ \\
\hline Total & 2.85 & 1.8 & 4.54 & 1.8 & 4.52 & 1.9 & 7.9 & $p<.01$ \\
\hline
\end{tabular}

Contraste de Bonferroni: Diferencias entre Cuento y Pasado, y entre Cuento y Futuro $(p<.01)$ 


\section{TABLA 7}

RECURSOS EVALUATIVOS MÁS UTILIZADOS POR LOS NIÑOS EN LAS NARRATIVAS PRODUCIDAS EN DIFERENTES CONTEXTOS CONVERSACIONALES

\begin{tabular}{|c|c|c|c|c|c|c|}
\hline \multirow{2}{*}{$\begin{array}{c}\text { Recursos más } \\
\text { utilizados }\end{array}$} & \multicolumn{2}{|c|}{ Cuento } & \multicolumn{2}{|c|}{ Pasado } & \multicolumn{2}{|c|}{ Futuro } \\
\hline & $f$ & $\%$ & $f$ & $\%$ & $f$ & $\%$ \\
\hline Irrealis & & & 34 & 27 & 21 & 25 \\
\hline Conector causal & 27 & 21 & & & & \\
\hline Evento interno & & & 28 & 22 & 28 & 33 \\
\hline
\end{tabular}

TABLA 8

RECURSOS EVALUATIVOS MÁS UTILIZADOS POR LOS INTERLOCUTORES EN LAS NARRATIVAS PRODUCIDAS EN DIFERENTES CONTEXTOS CONVERSACIONALES

\begin{tabular}{|c|c|c|c|c|c|c|}
\hline \multirow{2}{*}{$\begin{array}{c}\text { Recursos más } \\
\text { utilizados }\end{array}$} & \multicolumn{2}{|c|}{ Cuento } & \multicolumn{2}{|c|}{ Pasado } & \multicolumn{2}{|c|}{ Futuro } \\
\hline & $f$ & $\%$ & $f$ & $\%$ & $f$ & $\%$ \\
\hline Evento interno & 52 & 28 & 75 & 32 & 105 & 33 \\
\hline Irrealis & 50 & 27 & & & 83 & 26 \\
\hline
\end{tabular}




\section{REFERENCIAS BIBLIOGRÁFICAS}

Arrúe, J. (2012). Los contextos de crianza de niños pequeños de entornos socioculturales diversos. Un estudio de las actividades, los conocimientos y las interacciones que configuran los contextos en los hogares [Upbringing context of young children from different social and cultural groups. A study of the activities, knowledges and interactions that shape the home environment]. Tesis Doctoral no publicada. Facultad de Filosofía y Letras, Universidad de Buenos Aires.

Aukrust, V.G. \& Snow, C.E. (1998). Narratives and explanations during mealtime conversations in Norway and the U.S. Language in Society, 27(2), 221-246. http://dx. doi.org/10.1 017/s0047404500019862

Bamberg, M. \& Damrad-Frye, R. (1991). On the ability to provide evaluative comments. Further explorations of children's narrative competencies. Journal of Child Language, 18, 689710. http://dx.doi.org/10.1017/S0305000900 011314

Bamberg, M. \& Reilly, J.S. (1994). Emotion, narrative and affect: How children discover the relationship between what to say and how to say it. En D. Slobin, J. Gerhardt, A. Kyratzis \& J. Guo (Eds.), Interaction, social context and language: Essays in honor of Susan ErvinTripp (pp. 329-341). Hillsdale, NJ: Erlbaum.

Beck, S. (2008). Cultural variation in narrative competence and its implications for children's academic success. En A. McCabe, A. Bailey \& G. Melzi (Eds.), Spanish-language narration and literacy (pp. 332-350). Cambridge, UK: Cambridge University Press.

Bruner, J. (1986). El habla del niño [Child's talk]. Barcelona: Paidós.

Curenton, S.M., Craig, M.J. \& Flanigan, N. (2008). Use of decontextualized talk across story contexts: How oral storytelling and emergent reading can scaffold children's development. Early Education \& Development, 19 (1), 161-187. http://dx.doi.org/101080/10409 280701839296

Díaz Oyarce, C. \& Mendoza Saavedra, J. (2012). Evolución y progreso en el uso de estructuras evaluativas presentes en las producciones narrativas orales de niños y niñas de escuelas de sectores vulnerables [Evolution and progress in using evaluative structures present in the oral narrative productions of children in vulnerable schools]. Onomázein, 26(2), 391-410.

Eisenberg, A.R. (1985). Learning to describe past experience in conversation. Discourse Processes, 8, 177-204. http://dx.doi.org/10.1080/0 1638538509544613

Ely, R. Gleason, J.B. Narasimhan, B. \& McCabe, A. (1995). Family talk about talk: Mothers lead the way. Discourse Processes, 19, 201-218. http://dx.doi.org/10.1080/01638539509544914

Fernández, C. \& Melzi, G. (2008). Evaluation in Spanish-speaking mother-child narratives: The social and sense-making function of internalstate references. En A. McCabe, A. Bailey \& G. Melzi (Eds.), Spanish-language narration and literacy (pp. 92-118). Cambridge, UK: Cambridge University Press.

Fivush, R. (1991). The social construction of personal narratives. Merril-Palmer Quarterly, 37, 59-82.

Fivush, R. \& Nelson, K. (2006). Parent-child reminiscing locates the self in the past. British Journal of Developmental Psychology, 24, 235-251. http://dx.doi.org/10.1348/02615100 $5 \times 57747$

Haden, C.A., Haine, R.A. \& Fivush, R. (1997). Developing narrative structure in parent-child reminiscing across the preschool years. Developmental Psychology, 33(2), 295-307. http:// dx.doi.org/10.1037//0012-1469.33.2.295 
Hudson, J.A. (2002). Do you know what we're going to do this summer? Mothers' talk to preschool children about future events. Journal of Cognitive Development, 3, 49-71. http://dx. doi.org/10.1207/S15327647/JCD0301-4

Hudson, J.A. (2006). The development of future time concepts through mother-child conversation. Merrill-Palmer Quarterly, 52(1), 70-95. http://dx.doi.org/10.1353/mpq.2006.0 005

Kitamura, S. (1998). Ardilla tiene hambre [Squirrel is hungry]. Buenos Aires: Fondo de Cultura Económica.

Labov, W. (1972). Sociolinguistic patterns. Philadelphia, PA: University of Pennsylvania Press.

Labov, W. (2001). Uncovering the event structure of narrative. En D. Tannen \& J. Alatis. (Eds.), Georgetown University Roundtable on Language and Linguistics 2001 (pp. 63-83). Washington, DC: Georgetown University Press.

Labov, W. \& Waletzky, J. (1997). Narrative analysis: Oral versions of personal experience. Journal of Narrative of Life and History, 7(3), 3-38. (Trabajo original publicado en 1967).

Miller, P.J. \& Sperry, L.L. (1988). Early talk about the past: The origins of conversational stories of personal experiences. Journal of Child Language, 15, 293-315. http://dx.doi.org/10.1017/ S0305000900012381

Nelson, K. (1996). Language in cognitive development. Cambridge: Cambridge University Press.

Nelson, K. (2007). Young minds in social worlds. Experience, meaning and memory. Cambridge: Harvard University Press.

Nelson, K. \& Fivush, R. (2004). The emergence of autobiographical memory: A social-cultural developmental theory. Psychological Review, 111(2), 486-511. http://dx.doi.org/10.1037/003 3-295x.111.2.486

Newcombe, R. \& Reese, E. (2004). Evaluations and orientations in mother-child narratives as a function of attachment security: A longitudinal investigation. International Journal of Behavioral Development, 28(3), 230-245. http: //dx.doi.org/10.1080/01650250344000460

Pellegrini, A.D. (1985). The narrative organization of children's fantasy play. Educational Psychology, 5, 17-25. http://dx.doi.org/10.1080/0 144341850050103

Peterson, C. \& Biggs, M. (2001). "I was really, really, really mad!" Children's use of evaluative devices in narratives about emotional events. Sex Roles, 45(11-12), 801-825. http://dx.doi. org/10.1023/a:1015692403932

Peterson, C. \& McCabe, A. (1992). Parental style of narrative elicitation: Effect on children's narrative structure and content. First Language, 12, 299-321. http://dx.doi.org/10.1177 /014272379201203606

Peterson, C. \& McCabe, A. (1994). A social interactionist account of developing decontextualized narrative skill. Developmental Psychology, 30, 937-948. http://dx.doi.org/10.10 37/0012-1649.30.6.937

Romero Contreras, S. \& Gómez Martínez, G.E. (2013). El desarrollo del lenguaje evaluativo en narraciones de niños mexicanos de 3 a 12 años [Evaluative language development in narratives of Mexican children from 3 to 12 years]. Actualidades en Psicología, 27, 15-30.

Ryner, S. (2006). Cat and dog in a mess. London: Harper Collins.

Rosemberg, C.R., Silva, M.L. \& Stein, A. (2010). Narrativas infantiles en contexto: Un estudio en hogares de barrios urbano marginados de Buenos Aires. Revista del Instituto de Investigaciones en Ciencias de la Educación, 28, 135-154.

Shiro, M. (2003). Genre and evaluation in narrative development. Journal of Child Language, 30, 165-195. http://dx.doi.org/10.1017/S0305 000902005500 
Snow, C.E., Porche, M.V., Tabors, P.O. \& Harris, S.S. (2007). Is literacy enough? Pathways to academic success for adolescents. Illinois: Brookes.

Tomasello, M. (1999). The cultural origins of human cognition. Cambridge: Harvard University Press.

Tomasello, M. (2003). Constructing a language: A usage-based theory of language acquisition. Massachusetts: Harvard University Press.

Uccelli, P. (2009). Emerging temporality: Paste tense and temporal / aspectual markers in
Spanish-speaking children's intra-conversational narratives. Journal of Child Language, 36, 929-966. http://dx.doi.org/10.1017/S03050 00908009288

Vasilachis de Gialdino, I. (2006) (Coord.). Estrategias de investigación cualitativa [Qualitative research strategies]. Buenos Aires: Gedisa.

Véliz, M. (1988). Evaluación de la madurez sintáctica en el discurso escrito [Evaluation of syntactic maturity in written discourse]. Revista de Lingüistica Teórica y Aplicada, 26, 105-141.

Centro Interdisciplinario de Investigaciones en Psicología Matemática y Experimental Dr. Horacio J.A. Rimoldi (CIIPME) Consejo Nacional de Investigaciones Cientificas y Técnicas (CONICET) Ciudad Autónoma de Buenos Aires República Argentina

Fecha de recepción: 13 de mayo de 2014 Fecha de aceptación: 30 de septiembre de 2014 
